\title{
RISK FACTORS FOR VENOUS THROMBOEMBOLISM AFTER PEDIATRIC
}

\section{TRAUMA}

Casey J. Allen ${ }^{1}$, Clark R. Murray ${ }^{1}$, Jonathan P Meizoso ${ }^{1}$, Juliet J Ray ${ }^{1}$, Holly L. Neville ${ }^{2}$, Carl I. Schulman ${ }^{1}$, Nicholas Namias ${ }^{1}$, Juan E. Sola ${ }^{2}$, and Kenneth G. Proctor ${ }^{1}$

${ }^{1}$ Division of Trauma Surgery, ${ }^{2}$ Division of Pediatric Surgery, Daughtry Family Department of Surgery, University of Miami Miller School of Medicine, Miami, Florida, USA

Supported in part by: Grants \#N140610670 from the Office of Naval Research and \#09078015 from U.S. Army Medical Research \& Materiel Command

None of the authors have declared conflicts of interest.

Corresponding Author:

Kenneth G. Proctor, Ph.D.

Professor of Surgery

Divisions of Trauma and Surgical Critical Care

Daughtry Family Department of Surgery

University of Miami Miller School of Medicine, Ryder Trauma Center

1800 NW 10th Ave. Miami, FL 33136

Telephone: 305-585-1178 (office)

Fax: 305-326-7065

E-Mail: kproctor@miami.edu

SHORT TITLE: VTE in Pediatric Trauma

Level III Evidence 


\section{ABSTRACT}

BACKGROUND/PURPOSE: The purposes of this study were to identify independent predictors of venous thromboembolism (VTE), to evaluate the relative impact of adult VTE risk factors, and to identify a population at high-risk for VTE after pediatric trauma.

METHODS: 1,934 consecutive pediatric admissions ( $\leq 17$ years) from $01 / 2000$ to $12 / 2012$ at a level 1 trauma center were reviewed. Logistic regression was used to identify predictors of VTE.

RESULTS: Twenty-two patients (1.2\%) developed a VTE, including 5\% of those requiring orthopedic surgery, $14 \%$ of those with major vascular injury (MVI), and 36\% of those with both. Most (84\%) were diagnosed at the primary site of injury. $86 \%$ of those who developed a VTE were receiving thromboprophylaxis at the time of diagnosis. Independent predictors were age (odds ratio (OR): 1.59, 95\% confidence interval (CI): 1.11-2.25), orthopedic surgery (OR: 8.10, CI: 3.10-21.39), transfusion (OR: 3.37, CI: 1.26-8.99), and vascular surgery (OR: 15.43, CI: 5.70-41.76). When known risk factors for VTE in adults were adjusted, significant factors were age $\geq 13$ years (OR: 9.16, CI: 1.08-77.89), indwelling central venous catheter (CVC) (OR: 4.41, CI: 1.31-14.82), orthopedic surgery (OR: 6.80, CI: 2.47-18.74), and MVI (OR: 14.41, CI: 4.6045.13).

CONCLUSION: MVI and orthopedic surgery are synergistic predictors of pediatric VTE. Most children who developed a VTE were receiving thromboprophylaxis at the time of diagnosis.

Keywords: deep vein thrombosis, DVT, trauma, pediatrics, children, VTE 


\section{INTRODUCTION}

Trauma remains the leading cause of morbidity and mortality in children and adolescents $(1,2)$. Although traumatic injury induces a state of hypercoagulability in both adult (3) and pediatric (4) patients, venous thromboembolism (VTE) rates are significantly lower in children (5-13) yet carry significant morbidity (14). Although risk factors in the adult population are established (15-20), the factors leading to VTE in children are not clear. Therefore, the purposes of this study are to identify the independent predictors of VTE after pediatric trauma, to assess the relative impact of known risk factors for VTE in adult trauma on the pediatric population, and to identify a population at high-risk for VTE in children.

\section{METHODS}

After approval from the University of Miami Miller School of Medicine Institutional Review Board, consecutive pediatric admissions ( $\leq 17$ years old) from January 2000 to December 2012 at the Ryder Trauma Center in Miami, Florida were retrospectively reviewed. Those pregnant, incarcerated or not admitted to either the trauma or pediatric surgery services were excluded from analysis.

Demographics, mechanisms of injury (MOI), injuries sustained, interventions performed, initial vital signs and laboratory values, length of stay (LOS), and survival were reviewed. Hypotension and tachycardia were defined as any value outside of normal age-specific range for the child. Major vascular injury (MVI) was defined as any injury to a named vessel. Patient injuries and

other diagnoses, including obesity, were identified with use of International Classification of Diseases, Ninth Revision (ICD-9) coding. 
Adult risk factors for VTE included advanced age, obesity, head injury, MVI, indwelling central venous catheter (CVC), operation, orthopedic injury, spinal cord injury (SCI), pelvic fracture, and transfusion. An ISS > 15 was used to define a patient who was "severely injured".

VTE is defined as either a deep vein thrombosis (DVT) or pulmonary embolism (PE). The diagnosis of DVT and PE was obtained from ICD-9 coding and confirmed upon review of physician notes and radiology reports. No routine surveillance for DVT was performed. Thromboprophylaxis was given at the physician's discretion, and the agent used was as per institutional standards and pharmacy formulary during the study period. As children > 13 years of age are managed by the trauma surgery service at our institution, these patients typically receive thromboprophylaxis following injury, such as do the adult patients. Methods of thromboprophylaxis were reviewed and included low-dose unfractionated heparin (UFH; 5,000U subcutaneously three times daily) or low molecular weight heparin (LMWH; either dalteparin 5,000U subcutaneously once daily, or Enoxaparin 30mg subcutaneously once daily) as guided by the hospital formulary. In addition, sequential compression devices were used if not prohibited by plaster immobilizers or external fixators.

Statistical analyses were performed using SPSS version 22 (IBM Corporation; Armonk, NY). Parametric data are reported as mean \pm standard deviation and non-parametric data are reported as median (interquartile range). Data were compared using a t-test or Mann Whitney U test, as appropriate. Categorical variables were compared using a chi-square or Fisher's exact test, as appropriate. The results of the univariate analyses were used to include variables of significant 
association in a stepwise logistic regression model to identify the independent predictors of VTE. In addition, known risk factors for VTE in adults were included in a regression analysis to identify the adjusted risk factors in children. Statistical significance was determined at alpha level 0.05 .

\section{RESULTS}

The study population was comprised of 1,934 pediatric patients (Table 1). VTE developed in $1.2 \%(22 / 1,934)$ of the overall population. LOS was $3(1-8)$ days, and mortality was $3.5 \%$. VTE occurred in $5 \%$ of those requiring orthopedic surgery, $14 \%$ of those with MVI, and $36 \%$ of those with both. Most (84\%) VTE were diagnosed at the primary site of injury compared to $16 \%$ at an uninjured site. Three patients $(0.2 \%)$ developed a PE, yet none were concomitantly diagnosed with DVT (negative lower extremity venous duplex ultrasounds). Of those diagnosed with VTE, the majority was actively receiving thromboprophylaxis at time of diagnosis (86\%). Methods of thromboprophylaxis included UFH (42\%) and LMWH (58\%).

Of those who developed a DVT, the majority (10/19) sustained MVI. Of these, two did not receive thromboprophylaxis, six received some form of thromboprophylaxis, and 2 were therapeutically anticoagulated. Most of the MVI were not repaired but were rather ligated due to the complexity of the injury or the hemodynamic instability of the patient at time of surgery.

Table 2 compares patients with VTE to those without. In general, VTE patients were older, had higher injury severity scores (ISS) and worse hemodynamics. They more often had MVI, required orthopedic surgery, and had longer LOS. 
Variables of significant association (age, race, BE, age-specific hypotension, tachycardia, ISS, operation, MVI, orthopedic surgery, CVC, transfusion) were included in a stepwise logistic regression model. Independent predictors of VTE were identified as age (odds ratio (OR): 1.59, 95\% confidence interval (CI): 1.11-2.25), orthopedic surgery (OR: 8.10, CI: 3.10-21.39), transfusion (OR: 3.37, CI: 1.26-8.99), and MVI (OR: 15.43, CI: 5.70-41.76); area under receiver operator curve (AUROC): 0.939.

Known associated risk factors for adult VTE were included in a separate regression analysis. The significant adjusted risk factors were identified as age $\geq 13$ years (OR: 9.16, CI: 1.08-77.89), indwelling CVC (OR: 4.41, CI: 1.31-14.82), orthopedic surgery (OR: 6.80, CI: 2.47-18.74), and MVI (OR: 14.41, CI: 4.60-45.13); AUROC: 0.962 (Figure 1).

Within this population, when the DVT was at the primary site of injury were excluded, two patients had a CVC and DVT. Of these, one had a hemodialysis catheter placed after DVT was diagnosed, and the other patient had a right femoral CVC placed at admission and subsequently developed a DVT at the right common femoral vein.

When comparing risk factors of PE versus DVT, no associations were made likely due to low sample size. Still, those who developed a PE alone (no concomitant DVT) were all blunt trauma victims with severe injury severity, presented with hemodynamic instability at admission, required central venous access, blood transfusions and operative intervention. Ultimately, their clinical course was prolonged (average LOS 29d). None of the PE patients sustained MVI. All 
were actively receiving UFH at time of diagnosis, and, upon recognition, an inferior vena cava filter was placed.

\section{DISCUSSION}

The major findings of the study are that MVI and orthopedic surgery are synergistic predictors of DVT, the vast majority of all DVT occurred at the primary site of injury, and the independent predictors of VTE after pediatric trauma population are age, orthopedic surgery, MVI, and transfusion. When adjusting for the known risk factors for VTE in adults, MVI and orthopedic surgery were the greatest risk factors in children. Most children (86\%) who developed a VTE were receiving thromboprophylaxis at the time of diagnosis.

Age remains an important risk factor for VTE. The incidence of VTE in the pediatric trauma population ranges from $0.02 \%$ to $0.33 \%(1-8)$ while the rate of VTE in the adult trauma population is $6 \%$ to $7 \%(21,22)$. As with our analysis, the risk of VTE varies with age. Van Arendonk et al. found that the risk of VTE was lower among younger patients with an incidence of $0.1 \%$ in patients 12 years or younger (23). Young age has a protective role in developing VTE (24-31) probably because the vascular endothelium of children has not accumulated the extensive damage seen in adults from various diseases (32).

Ryan et al. demonstrated that pediatric trauma patients (4) have a similar state of hypercoagulability as adult trauma patients (33). Despite this, DVT is rare (14), thus making it difficult to identify a pediatric cohort at high-risk. From our findings, when both MVI and orthopedic surgery were present, DVT occurred in over one-third of patients. Although no single 
factor is necessary or sufficient for the pathogenesis of DVT (hypercoagulability, etc.); in general, the condition in children may depend more on direct endothelial cell damage and stasis than that of a hypercoagulable state.

Currently, no national standardized guidelines exist for VTE prophylaxis after pediatric trauma, but the rarity of VTE in pediatric trauma patients has led many surgeons to conclude that pharmacologic VTE prophylaxis is unnecessary. There is little doubt that the relative risk of VTE after trauma is considerably lower in the pediatric versus the adult population, but the precise age at which the risk of VTE increases is unclear (23). In fact, practices vary considerably in adolescent trauma patients (23). A survey of 133 US trauma centers found that for inpatients aged 11 to 15 years, $13 \%$ of trauma surgeons use low molecular weight heparin (LMWH) often or always, 25\% sometimes, and 62\% rarely or never (34). Even within our institution, practices vary according to physician preference. For these reasons, we performed an in-depth analysis of our population. Our findings identify a specific high-risk in children: that at the site of combined major vascular and orthopedic injury.

There is ongoing investigation on the efficacy of VTE prophylaxis in the high-risk population. Despite standard prophylaxis, high rates of VTE persist in adults (35). Since our findings showed that most children (86\%) who developed a VTE were receiving thromboprophylaxis at the time of diagnosis., it is difficult to assess its efficacy. The majority of children over the age of 13 years received some form of thromboprophylaxis, yet this is the population at high independent risk for VTE. Perhaps in some of these patients, VTE could have been prevented if therapeutically anticoagulated (even though two patients who were anticoagulated still developed 
a DVT), or perhaps regardless of treatment these patients are still at risk for development of VTE. However, this was not the direct purpose of our study, rather it was to identify a population at high-risk for VTE and to identify what injury factors were associated.

The association between DVT and PE after penetrating trauma is well documented (36). It is generally assumed that PE likely results from a propagated DVT at the site of isolated injury. In this present study, none of the patients who developed a PE were diagnosed with DVT. There is recent evidence that a primary pulmonary thrombus can arise without evidence of DVT following severe blunt trauma (37-40). This has been shown to be clinically distinct from PE and is more prevalent and symptomatic than traditional PE developing from the deep venous system (41). Our findings appear to support this theory of separate disease processes; that of PE being in those with severe blunt trauma, critical illness, prolonged immobility, etc., as opposed to major vascular and orthopedic injury at site of DVT.

There are limitations to this study. This data were collected retrospectively and at a single institution. Some variables may have been missing or misclassified. This study population was comprised only of patients admitted to a trauma center, so it cannot be generalized to those discharged home from the emergency department with lesser injuries. Post-operative thromboprophylaxis was also based on physician discretion. No protocol was in place to follow patients for the development of any VTE; appropriate diagnostic tests were ordered according to clinical suspicion. It is possible that sub-clinical DVT or PE may have occurred without diagnosis. Patients with DVT were obtained from a query of our trauma database with use of ICD-9 coding, and thus we may have unintentionally excluded patients that had DVT 
undiagnosed by these means. As recent large population studies analyze pediatric DVT rates with use of ICD-9 coding (42), it was our objective to assess the development of clinical DVT diagnosed by these means and to further investigate these diagnoses upon thorough review of patient injury patterns, location of DVT, and hospital course.

In summary, within the context of these limitations, MVI and orthopedic surgery are synergistic predictors of DVT, the vast majority of all DVT occurred at the primary site of injury, and the independent predictors of VTE in our pediatric trauma population are age, orthopedic surgery, MVI, and transfusion. MVI and orthopedic surgery were the greatest risk factors in children. And finally, most children (86\%) who developed a VTE were receiving thromboprophylaxis at the time of diagnosis. These findings contribute to the growing body of knowledge on this topic, providing a set of injury factors that can identify children at high-risk that may benefit from more aggressive preventive therapies.

\section{ACKNOWLEDGEMENTS:}

We thank Jonathan Andron for his help and assistance in maintaining the trauma registry.

\section{AUTHOR CONTRIBUTION:}

CJA is directly responsible for all aspects of this study. He participated in the conception and experimental design; collection, analysis and interpretation of data; drafting and revision of the manuscript, figures and tables.

CRM, JPM, and JJR participated in the conception and experimental design; analysis and interpretation of data; revision of the manuscript, figures and tables. 
HLN, CIS, NN, and JES were medically responsible for a fraction of the patients in this study. In addition, they participated in the conception and experimental design; revision of the manuscript, figures and tables.

KGP had overall responsibility for the study; including conception and experimental design; analysis and interpretation of data; drafting and revision of the manuscript, figures and tables; statistical expertise and evaluation; supervision.

\section{REFERENCES}

1. Gratz RR. Accidental injury in childhood: a literature review on pediatric trauma. J Trauma 1979;19(8):551-5.

2. Rivara FP. Epidemiology of violent deaths in children and adolescents in the United States. Pediatrician 1983;12(1):3-10.

3. Van Haren RM, Valle EJ, Thorson CM, Jouria JM, Busko AM, Guarch GA, et al. Hypercoagulability and other risk factors in trauma intensive care unit patients with venous thromboembolism. J Trauma. 2014;76(2):443-9.

4. Ryan ML, Van Haren RM, Thorson CM, Andrews DM, Perez EA, Neville HI, Sola JE et al. Trauma Induced hypercoagulability in pediatric patients. J Pediatr Surg. 49 (2014) 1295-9.

5. Candrilli SD, Balkrishnan R, O'Brien SH. Effect of injury severity on the incidence and utilization-related outcomes of venous thromboembolism in pediatric trauma inpatients. Pediatr Crit Care Med 2009; 10(5):554-7.

6. Askegard-Giesmann JR, O'Brien SH, Wang W, et al. Increased use of enoxaparin in pediatric trauma patients. J Pediatr Surg 2012;47(5):980-3. 
7. McBride WJ, Gadowski GR, Keller MS, Vane DW. Pulmonary embolism in pediatric trauma patients. J Trauma 1994;37(6):913-5.

8. Cook A, Shackford S, Osler T, Rogers F, Sartorelli K, Littenberg G. Use of vena cava filters in pediatric trauma patients: data from the National Trauma Data Bank. J Trauma 2005;59(5):1114-20.

9. Cyr C, Michon B, Pettersen G, David M, Brossard J. Venous thromboembolism after severe injury in children. Acta Haematol 2006;115(3-4): 198-200.

10. O'Brien SH, Candrilli SD. In the absence of a central venous catheter, risk of venous thromboembolism is low in critically injured children, adolescents, and young adults: evidence from the National Trauma Data Bank. Pediatr Crit Care Med 2011;12(3):251-6.

11. Truitt AK, Sorrells DL, Halvorson E, Starring J, Kurkchubasche AG, Tracy TF Jr, et al. Pulmonary embolism: which pediatric trauma patients are at risk? J Pediatr Surg 2005;40(1):1247.

12. Vavilala MS, Nathens AB, Jurkovich GJ, Mackenzie E, Ravara FP. Risk factors for venous thromboembolism in pediatric trauma. J Trauma 2002;52(5): 922-7.

13. Geerts WH, Code KI, Jay RM, Chen E, Szalai JP. A prospective study of venous thromboembolism after major trauma. The New England journal of medicine. 1994;331(24):1601-6.

14. Kenet G, Strauss T, Kaplinsky C, Paret G. Hemostasis and thrombosis in critically ill children. Semin Thromb Hemost 2008;34(5):451-8.

15. Haut ER, Chang DC, Pierce CA, et al: Predictors of posttraumatic deep vein thrombosis (DVT): Hospital practice versus patient factors-an analysis of the National Trauma Data Bank (NTDB). J Trauma 2009; 66:994-999 
16. Rogers FB, Cipolle MD, Velmahos G, et al: Practice management guidelines for the prevention of venous thromboembolism in trauma patients: The EAST practice management guidelines work group. J Trauma 2002; 53:142-164

17. Gearhart MM, Luchette FA, Proctor MC. et al: The risk assessment profile score identifies trauma patients at risk for deep vein thrombosis. Surgery 2000; 128:631-640

18. Greenfield LJ, Proctor MC, Rodriguez JL, Luchette FA, Cipolle MD, Cho J. Posttrauma thromboembolism prophylaxis. J Trauma. 1997; 42 (1): 100-103

19. Sharma OP, Oswanski MF, Joseph RJ, et al: Venous thromboembolism in trauma patients. Am Surg 2007; 73:1173-1180

20. Thorson CM, Ryan ML, Van Haren RM, Curia E, Barrera JM, Guarch GA, Busko AM, Namias N, Livingstone AS, Proctor KG. Venous thromboembolism after trauma: a never event? Crit Care Med. 2012; 40 (11): 2967-2973.

21. Shackford SR, Davis JW, Hollingsworth-Fridlund P, Brewer NS, Hoyt DB, Mackersie RC. Venous thromboembolism in patients with major trauma. Am J Surg. 1990;159(4):365-9.

22. Toker S, Hak DJ, Morgan SJ. Deep vein thrombosis prophylaxis in trauma patients. Thrombosis. 2011; 2011:505373.

23. Van Arendonk KJ, Schneider EB, Haider AH, Colombani PM, Stewart FD, Haut ER. Venous Thromboembolism After Trauma When do children Become Adults? JAMA Surg. 2013: 148(12): 1123-30.

24. Andrew M, Schmidt B, Mitchell L, Paes B, Ofosu F. Thrombin generation in newborn plasma is critically dependent on the concentration of prothrombin. Thromb Haemost 1990;63:27-30.

25. Andrew M, Mitchell L, Vegh P, Ofosu F. Thrombin regulation in children differs from 
adults in the absence and presence of heparin. Thromb Haemost 1994;72:836-42.

26. Ling X, Delorme M, Berry L, Ofosu F, Mitchell L, Paes B, et al. Alpha 2-macroglobulin remains as important as antithrombin III for thrombin regulation in cord plasma in the presence of endothelial cell surfaces. Pediatr Res 1995;37:373-8.

27. Delorme MA, Xu L, Berry L, Mitchell L, Andrew M. Anticoagulant dermatan sulfate proteoglycan (decorin) in the term human placenta. Thromb Res 1998;90:147-53.

28. Andrew M, Mitchell L, Berry L, Paes B, Delorme M, Ofosu F, et al. An anticoagulant dermatan sulfate proteoglycan circulates in the pregnant woman and her fetus. J Clin Invest 1992;89:321-6.

29. Delorme MA, Burrows RF, Ofosu FA, Andrew M. Thrombin regulation in mother and fetus during pregnancy. Semin Thromb Hemost 1992;18:81-90.

30. Nitschmann E, Berry L, Bridge S, Dereske M, Richardson M, Monagle P, et al. Morphologic and biochemical features affecting the antithrombotic properties of the inferior vena cava of rabbit pups and adult rabbits. Pediatr Res 1998;43:62-7.

31. Nitschmann E, Berry L, Bridge S, Hatton MW, Richardson M, Monagle P, et al. Morphological and biochemical features affecting the antithrombotic properties of the aorta in adult rabbits and rabbit pups. Thromb Haemost 1998;79:1034-40.

32. Spentzouris G, Scriven RJ, Lee TK, Labropoulos N. Pediatric Venous Thromboembolism in relation to adults. J Vasc Surg. 2012 Jun;55(6):1785-93

33. Schreiber MA, Differding J, Thorborg P, Mayberry JC, Mullins RJ. Hypercoagulability is most prevalent early after injury and in female patients. J Trauma 2005;58(3):475-80

34. O’Brien SH, Haley K, Kelleher KJ, WangW, McKenna C, Gaines BA. Variation in DVT prophylaxis for adolescent trauma patients: a survey of the Society of Trauma Nurses. J Trauma 
Nurs. 2008;15(2):53-57.

35. Allen CJ, Murray CM, Meizoso JP, Ray JJ, Ruiz XD, Hanna MM, Schulman CI, Ginzburg E, Namias N, Proctor KG. Coagulation Profile Changes Due to Thromboprophylaxis and Platelets in Trauma Patients at High-Risk For Venous Thromboembolism. Am Surg. 2015 Jul;81(7):663-8.

36. Huisman MV, Buller HR, ten Cate JW, van Royen EA, Vreeken J, Kersten MJ, Bakx B. Unexpected high prevalence of silent pulmonary embolism in patients with deep venous thrombosis. Chest. 1989;95(3):498-502.

37. Knudson MM, Gomez D, Haas B, Cohen MJ, Nathens AB. Three thousand seven hundred thirty-eight posttraumatic pulmonary emboli: a new look at an old disease. Ann Surg. 2011;254(4):625-32.

38. Velmahos GC, Spaniolas K, Tabbara M, Abujudeh HH, de Moya M, Gervasini A, Alam HB. Pulmonary embolism and deep venous thrombosis in trauma: are they related? Arch Surg. 2009;144(10):928-32.

39. Kahn SA, Schubmehl H, Stassen NA, Sangosanya A, Cheng JD, Gestring ML, Bankey PE. Risk factors associated with venous thromboembolism in isolated blunt chest trauma. Am Surg. 2013;79(5):502-5.

40. Schwartz T, Hingorani A, Ascher E, Marks N, Shiferson A, Jung D, Jimenez R, Jacob T. Pulmonary embolism without deep venous thrombosis. Ann Vas Surg. 2012;26(7):973-6.

41. Van Gent JM, Zander AL, Olson EJ, Shackford SR, Dunne CE, Sise CB, Badiee J, Schechter MS, Sise MJ. Pulmonary embolism without deep venous thrombosis: De novo or missed deep venous thrombosis? J Trauma Acute Care Surg. 2014;76(5):1270-4. 


\section{FIGURE AND TABLE LEGENDS}

Table 1: Population Demographics $(\mathrm{n}=1,934)$.

GCS= Glasgow Coma Score, ISS=injury severity score, MVI= major vascular injury, DVT=deep vein thrombosis, $\mathrm{PE}=$ pulmonary embolism, $\mathrm{LOS}=$ length of stay

Table 2: Comparison of Non-VTE vs VTE patients $(\mathrm{n}=1,934)$.

VTE: venous thromboembolism, GCS= Glasgow Coma Score, AIS= abbreviated injury score, ISS=injury severity score, $\mathrm{CVC}=$ central venous catheter, $\mathrm{MVI}=$ major vascular injury, LOS= length of stay.

Figure 1: Results of regression analysis; adjusted odds for VTE.

ISS: injury severity score, AIS: abbreviated injury score, GCS: Glasgow Coma Scale, CVC: central venous catheter, MVI=major vascular injury 
Table 1: Population Demographics $(n=1,934)$.

\begin{tabular}{|llr|}
\hline Age, years & & $11 \pm 6$ \\
\hline Gender & Male & $70 \%$ \\
& Female & $30 \%$ \\
\hline Race & Black & $50 \%$ \\
& Hispanic & $27 \%$ \\
& White & $23 \%$ \\
\hline Mechanism of Injury & Blunt & $76 \%$ \\
& Penetrating & $24 \%$ \\
\hline Base Excess, mEq/L & & $-3 \pm 5$ \\
\hline Hematocrit, \% & & $37 \pm 6$ \\
\hline Hypotension & & $6 \%$ \\
\hline Tachycardia & $32 \%$ \\
GCS & & $15(14-$ \\
& & $15)$ \\
Intracranial hemorrhage & & $4 \%$ \\
ISS & & $13 \pm 12$ \\
Transfusion & $11 \%$ \\
Operative Intervention & $22 \%$ \\
MVI & & $5 \%$ \\
Orthopedic Surgery & & $13 \%$ \\
DVT & & $1.0 \%$ \\
PE & & $0.2 \%$ \\
LOS, days & $3(1-8)$ \\
Mortality & & $3.5 \%$ \\
\hline
\end{tabular}

GCS= Glasgow Coma Score, ISS=injury severity score, MVI= major vascular injury, DVT=deep vein thrombosis, $\mathrm{PE}=$ pulmonary embolism, $\mathrm{LOS}=$ length of stay 
Table 2: Comparison of Non-VTE vs VTE patients $(n=1,934)$.

\begin{tabular}{|c|c|c|c|c|}
\hline \multicolumn{2}{|l|}{ Age, years } & $11 \pm 6$ & $16 \pm 1$ & $<0.001$ \\
\hline \multirow[t]{2}{*}{ Gender } & Male & $70 \%$ & $77 \%$ & 0.495 \\
\hline & Female & $31 \%$ & $23 \%$ & \\
\hline \multirow{4}{*}{ Race } & Black & $50 \%$ & $50 \%$ & 0.001 \\
\hline & Hispanic & $28 \%$ & $18 \%$ & \\
\hline & White & $23 \%$ & $27 \%$ & \\
\hline & Other & $0 \%$ & $5 \%$ & \\
\hline \multirow[t]{2}{*}{ Mechanism of Injury } & Blunt & $76 \%$ & $64 \%$ & 0.209 \\
\hline & Penetrating & $24 \%$ & $36 \%$ & \\
\hline \multicolumn{2}{|l|}{ Obesity } & $1 \%$ & $5 \%$ & 0.148 \\
\hline \multicolumn{2}{|l|}{ Base excess, mEq/L } & $-3 \pm 5$ & $-6 \pm 5$ & 0.008 \\
\hline \multicolumn{2}{|l|}{ Hematocrit, \% } & $37 \pm 5$ & $35 \pm 6$ & 0.146 \\
\hline \multicolumn{2}{|c|}{ Age-specific hypotension } & $6 \%$ & $29 \%$ & 0.001 \\
\hline \multicolumn{2}{|c|}{ Age-specific tachycardia } & $31 \%$ & $80 \%$ & 0.002 \\
\hline \multicolumn{2}{|c|}{ Abnormal coagulation } & $2 \%$ & $5 \%$ & 0.392 \\
\hline \multicolumn{2}{|l|}{ GCS } & $15(14-15)$ & $15(13-15)$ & 0.563 \\
\hline \multicolumn{2}{|c|}{ Intracranial hemorrhage } & $4 \%$ & $0 \%$ & 0.622 \\
\hline \multicolumn{2}{|l|}{ ISS } & $13 \pm 12$ & $23 \pm 15$ & $<0.001$ \\
\hline \multicolumn{2}{|l|}{ Indwelling CVC } & $4 \%$ & $36 \%$ & $<0.001$ \\
\hline \multicolumn{2}{|l|}{ Pelvic Fracture } & $9 \%$ & $14 \%$ & 0.454 \\
\hline \multicolumn{2}{|l|}{ Spinal Cord Injury } & $3 \%$ & $5 \%$ & 0.490 \\
\hline \multicolumn{2}{|l|}{ Transfusion } & $10 \%$ & $47 \%$ & $<0.001$ \\
\hline \multicolumn{2}{|l|}{ Operative Intervention } & $22 \%$ & $73 \%$ & $<0.001$ \\
\hline \multicolumn{2}{|l|}{ Major Vascular Injury } & $5 \%$ & $64 \%$ & $<0.001$ \\
\hline \multicolumn{2}{|l|}{ Orthopedic Surgery } & $13 \%$ & $64 \%$ & $<0.001$ \\
\hline \multicolumn{2}{|l|}{ LOS, days } & $3(1-7)$ & $26(14-42)$ & $<0.001$ \\
\hline \multicolumn{2}{|l|}{ Mortality } & $3.5 \%$ & $9.1 \%$ & 0.181 \\
\hline
\end{tabular}

VTE: venous thromboembolism, GCS= Glasgow Coma Score, AIS= abbreviated injury score, ISS=injury severity score, $\mathrm{CVC}=$ central venous catheter, $\mathrm{MVI}=$ major vascular injury, $\mathrm{LOS}=$ length of stay. 
Figure 1 - Results of regression analysis; adjusted odds for VTE $(\mathrm{n}=1,934)$.

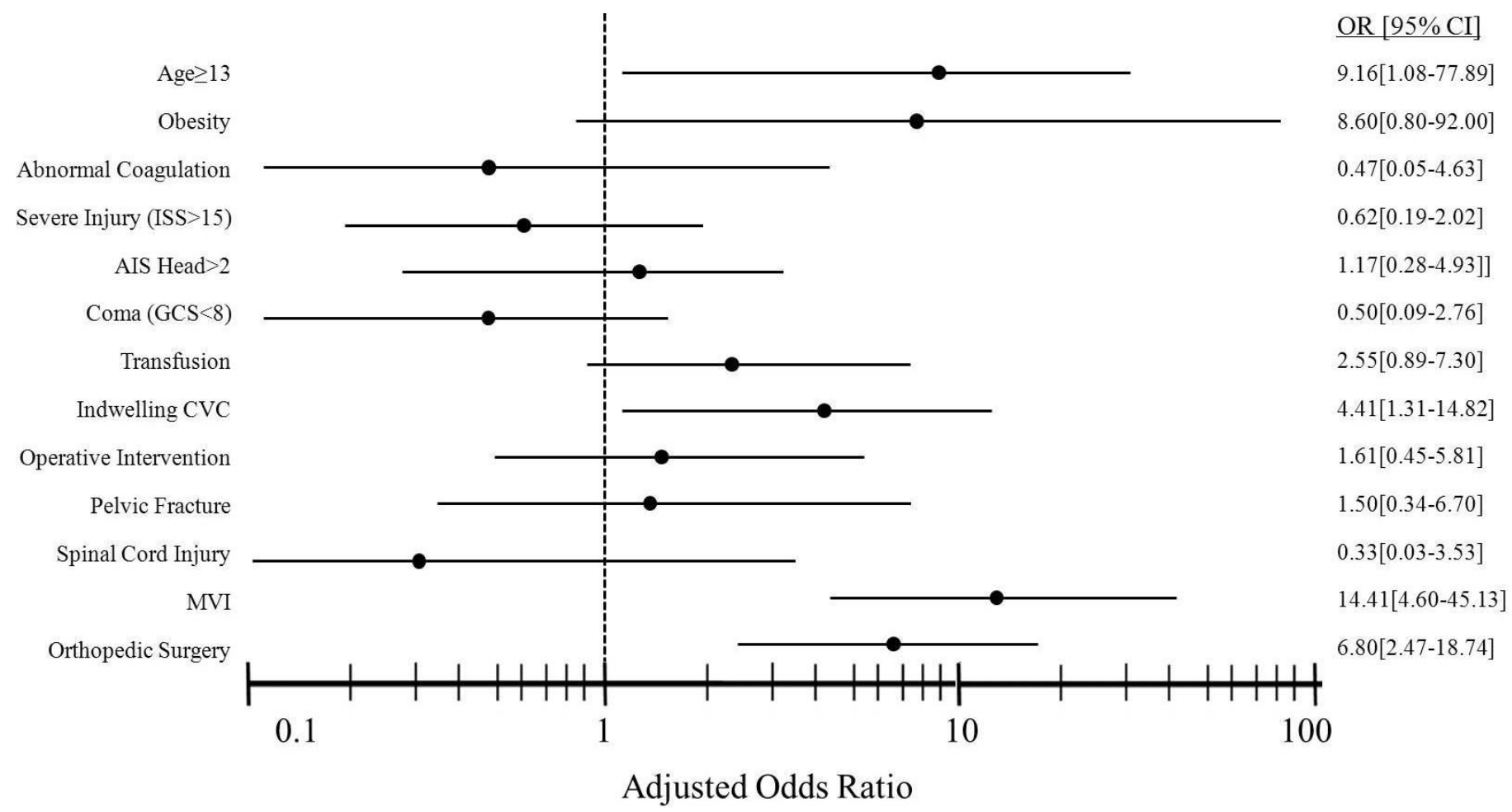

ISS: injury severity score, AIS: abbreviated injury score, GCS: Glasgow Coma Scale, CVC: central venous catheter, MVI=major vascular injury 